\title{
HETEROGENEITY AND BEHAVIORAL RESPONSE IN CONTINUOUS TIME CAPTURE-RECAPTURE, WITH APPLICATION TO STREET CANNABIS USE IN ITALY
}

\author{
By Alessio Farcomeni* and Daria Scacciatelli ${ }^{\dagger}$ \\ Sapienza - University of Rome* and University of Rome - Tor Vergata
}

We propose a general and flexible capture-recapture model in continuous time. Our model incorporates time-heterogeneity, observed and unobserved individual heterogeneity, and behavioral response to capture. Behavioral response can possibly have a delayed onset and a finite-time memory. Estimation of the population size is based on the conditional likelihood after use of the EM algorithm. We develop an application to the estimation of the number of adult cannabinoid users in Italy.

\section{APPENDIX A: FIRST DERIVATIVES OF THE ECCL}

We start giving an expression for the log-ECCL in terms of the functions $\mathrm{A}$ and $\mathrm{B}$ introduced:

$$
\begin{aligned}
l_{E C C L} & =\sum_{i=1}^{n}\left\{\ln f\left(\hat{\rho}_{i}\right)+\left(N_{i}(\tau)-1\right) \ln (\phi)+N_{i}(\tau) \ln \left(\gamma_{i}\right)+\sum_{j=1}^{N_{i}(\tau)}\left\{\ln \left(d N\left(t_{i j}\right)\right)\right.\right. \\
& \left.-\ln \left(B\left(\phi, \gamma, \hat{\rho}, \Omega(\tau), t_{i j}\right)\right)\right\}+N_{i}(\tau) \ln \left(\hat{\rho}_{i}\right) \\
& \left.-\hat{\rho}_{i} \gamma_{i}\left[\sum_{k} \phi^{I\left(t_{k}>t_{i 1}\right)} \frac{d N\left(t_{k}\right)}{B\left(\phi, \gamma, \hat{\rho}, \Omega(\tau), t_{k}\right)}+\Omega(\tau)\right]-\ln \left(A\left(\gamma_{i}, \hat{\rho}_{i}, \Omega(\tau)\right)\right)\right\} .
\end{aligned}
$$

The derivative of the log-ECCL with respect to $\phi$ can be obtained as:

$$
\begin{array}{r}
\frac{\partial l_{E C C L}}{\partial \phi}=\sum_{i=1}^{n}\left\{\frac{\left(N_{i}(\tau)-1\right)}{\phi}-\sum_{j=1}^{N_{i}(\tau)} \frac{1}{B\left(\phi, \gamma, \hat{\rho}, \Omega(\tau), t_{i j}\right)} \frac{\partial B\left(\phi, \gamma, \hat{\rho}, \Omega(\tau), t_{i j}\right)}{\partial \phi}\right. \\
\left.-\hat{\rho}_{i} \gamma_{i} \sum_{k}\left[I\left(t_{i 1}<t_{k}\right) \frac{d N\left(t_{k}\right)}{B\left(\phi, \gamma, \hat{\rho}, \Omega(\tau), t_{k}\right)}-\phi^{I\left(t_{i 1}<t_{k}\right)} \frac{d N\left(t_{k}\right)}{B\left(\phi, \gamma, \hat{\rho}, \Omega(\tau), t_{k}\right)^{2}} \frac{\partial B\left(\phi, \gamma, \hat{\rho}, \Omega(\tau), t_{k}\right)}{\partial \phi}\right]\right\}
\end{array}
$$

Keywords and phrases: behavioral response, capture-recapture, drug abuse, frailty, heterogeneity, Horvitz-Thompson estimator 
The derivative with respect to $\Omega(\tau)$ is instead:

$$
\begin{array}{r}
\frac{\partial l_{E C C L}}{\partial \Omega(\tau)}=\sum_{i=1}^{n}\left\{-\sum_{j=1}^{N_{i}(\tau)} \frac{1}{B\left(\phi, \gamma, \hat{\rho}, \Omega(\tau), t_{i j}\right)} \frac{\partial B\left(\phi, \gamma, \hat{\rho}, \Omega(\tau), t_{i j}\right)}{\partial \Omega(\tau)}\right. \\
\left.+\hat{\rho}_{i} \gamma_{i}\left[\sum_{k} \phi^{I\left(t_{i 1}<t_{k}\right)} \frac{d N\left(t_{k}\right)}{B\left(\phi, \gamma, \hat{\rho}, \Omega(\tau), t_{k}\right)^{2}} \frac{\partial B\left(\phi, \gamma, \hat{\rho}, \Omega(\tau), t_{k}\right)}{\partial \Omega(\tau)}-1\right]-\frac{1}{A\left(\gamma_{i}, \hat{\rho}_{i}, \Omega(\tau)\right)} \frac{\partial A\left(\gamma_{i}, \hat{\rho}_{i}, \Omega(\tau)\right)}{\partial \Omega(\tau)}\right\}
\end{array}
$$

Finally, for $s=1, \ldots, p$, the derivative with respect to $\beta_{s}$ is:

$$
\frac{\partial l_{E C C L}}{\partial \beta_{s}}=\sum_{h=1}^{n} \frac{\partial l_{E C C L}}{\partial \gamma_{h}} \frac{\partial \gamma_{h}}{\partial \beta_{s}}=\sum_{h=1}^{n} \frac{\partial l_{E C C L}}{\partial \gamma_{h}} \gamma_{h} Z_{h, s} ;
$$

where for $h=1, \ldots, n$

$$
\begin{array}{r}
\frac{\partial l_{E C C L}}{\partial \gamma_{h}}=\frac{N_{h}(\tau)}{\gamma_{h}}-\sum_{i=1}^{n}\left\{\sum_{j=1}^{N_{i}(\tau)} \frac{1}{\left.B\left(\phi, \gamma, \hat{\rho}, \Omega(\tau), t_{i j}\right)\right)} \frac{\left.\partial B\left(\phi, \gamma, \hat{\rho}, \Omega(\tau), t_{i j}\right)\right)}{\partial \gamma_{h}}\right. \\
\left.-\hat{\rho}_{i} \gamma_{i}\left[\sum_{k} \phi^{I\left(t_{i 1}<t_{k}\right)} \cdot \frac{d N\left(t_{k}\right)}{B\left(\phi, \gamma, \hat{\rho}, \Omega(\tau), t_{k}\right)^{2}} \frac{\partial B\left(\phi, \gamma, \hat{\rho}, \Omega(\tau), t_{k}\right)}{\partial \gamma_{h}}\right]+\frac{1}{A\left(\gamma_{i}, \hat{\rho}_{i}, \Omega(\tau)\right)} \frac{\partial A\left(\gamma_{i}, \hat{\rho}_{i}, \Omega(\tau)\right)}{\partial \gamma_{h}}\right\} \\
-\hat{\rho}_{h}\left[\sum_{k} \phi^{I\left(t_{h 1}<t_{k}\right)} \frac{d N\left(t_{k}\right)}{B\left(\phi, \gamma, \hat{\rho}, \Omega(\tau), t_{k}\right)}+\Omega(\tau)\right] .
\end{array}
$$

It is now a matter of obtaining a closed form expressions for the partial derivatives of $A$ and $B$. For what concerns the derivatives with respect to $\Omega(\tau)$, we have

$$
\begin{aligned}
\frac{\partial A\left(\gamma_{h}, \hat{\rho}_{h}, \Omega(\tau)\right)}{\partial \Omega(\tau)} & =-\hat{\rho}_{h} \gamma_{h} e^{\hat{\rho}_{h} \gamma_{h} \Omega(\tau)} A\left(\gamma_{h}, \hat{\rho}_{h}, \Omega(\tau)\right)^{2} ; \\
\frac{\partial B\left(\phi, \gamma, \hat{\rho}, \Omega(\tau), t_{k}\right)}{\partial \Omega(\tau)} & =\sum_{h=1}^{n} \hat{\rho}_{h} \gamma_{h} \frac{\partial A\left(\gamma_{h}, \hat{\rho}_{h}, \Omega(\tau)\right)}{\partial \Omega(\tau)} ;
\end{aligned}
$$

while for what concerns $\phi$,

$$
\begin{aligned}
\frac{\partial A\left(\gamma_{h}, \hat{\rho}_{h}, \Omega(\tau)\right)}{\partial \phi} & =0 \\
\frac{\partial B\left(\phi, \gamma, \hat{\rho}, \Omega(\tau), t_{k}\right)}{\partial \phi} & =\sum_{h=1}^{n} \hat{\rho}_{h} \cdot \gamma_{h} \cdot I\left(t_{h 1}<t_{k}\right) .
\end{aligned}
$$

We only need now an expression for the partial derivatives of $A$ and $B$ with respect to $\beta_{h} h=1, \ldots, p$. These are obtained as

$$
\begin{aligned}
\frac{\partial A\left(\gamma_{h}, \hat{\rho}_{h}, \Omega(\tau)\right)}{\partial \gamma_{h}}=-\hat{\rho}_{h} \Omega(\tau) \cdot e^{\hat{\rho}_{h} \cdot \gamma_{h} \cdot \Omega(\tau)} \cdot A\left(\gamma_{h}, \hat{\rho}_{h}, \Omega(\tau)\right)^{2} ; \\
\frac{\partial B\left(\phi, \gamma, \hat{\rho}, \Omega(\tau), t_{k}\right)}{\partial \gamma_{h}}=\hat{\rho}_{h} \cdot\left[\phi^{I\left(t_{h 1}<t_{k}\right)}+A\left(\gamma_{h}, \hat{\rho}_{h}, \Omega(\tau)\right)\right]+\hat{\rho}_{h} \gamma_{h} \frac{\partial A\left(\gamma_{h}, \hat{\rho}_{h}, \Omega(\tau)\right)}{\partial \gamma_{h}} .
\end{aligned}
$$


After plug in of the partial derivatives of $A$ and $B$ in the partial derivatives of $l_{E C C L}$, and straightforward algebra, we obtain the expressions stated in the main paper.

SAPIENZA - University OF Rome

Piazzale Aldo Moro, 5

00185 Rome

ITALY
University of Rome - TOR Vergata Via Orazio Raimondo 18

00173 RoME

ITALY 